\title{
Automated detection of Martian water ice clouds using Support Vector Machine and simple feature vectors
}

\author{
Kazunori Ogohara $^{\mathrm{a}, *}$, Takafumi Munetomo ${ }^{\mathrm{a}}$, Yuji Hatanaka ${ }^{\mathrm{a}}$, Susumu Okumura ${ }^{\mathrm{a}}$ \\ ${ }^{a}$ University of Shiga Prefecture, 2500 Hassaka, Hikone, Shiga, Japan
}

\begin{abstract}
We present a method for evaluating the presence of Martian water ice clouds using difference images and crosscorrelation distributions calculated from blue band images of the Valles Marineris obtained by the Mars Orbiter Camera onboard the Mars Global Surveyor (MGS/MOC). We derived one subtracted image and one cross-correlation distribution from two reflectance images. The difference between the maximum and the average, variance, kurtosis, and skewness of the subtracted image were calculated. Those of the cross-correlation distribution were also calculated. These eight statistics were used as feature vectors for training Support Vector Machine because they were the simplest of features that was expected to be closely associated with the physical properties of water ice clouds. The generalization ability was tested using 10 -fold cross-validation. F-measure and accuracy tended to be approximately 0.8 if the maximum in the normalized reflectance and the difference of the maximum and the average in the crosscorrelation were selected as features. This result can be physically explained because the blue band as well as the red band is sensitive to water ice clouds. A simple and low-dimensional feature vector enables us to understand the detected water ice clouds physically and presents the lower bound of the score that classifiers trained using more sophisticated feature vectors have to achieve.
\end{abstract}

Keywords: Mars, water ice cloud, automated detection, Mars Global Surveyor, machine learning

\section{Introduction}

Although it has been recently suggested that there may be liquid water on the present surface of Mars, Martian 3 water ice clouds were already identified decades ago (Martín-Torres et al., 2015). Wang and Ingersoll (2002) vi4 sually detected water ice clouds on images taken by the Mars Orbiter Camera onboard the Mars Global Surveyor 5 (MGS/MOC) and revealed that water ice clouds frequently occur in low latitudes and near mountains. Some general 6 circulation models (GCM) have successfully generated water ice clouds in such regions and have shown that wa7 ter ice clouds have an important role in water circulation in the Martian atmosphere and surface (Richardson, 2002, 8 Montmessin et al., 2004, Forget et al. 2006, Wilson et al., 2007). It has been revealed using mesoscale numerical

\footnotetext{
${ }^{*}$ Corresponding author

Email address: ogohara.k@e.usp.ac.jp (Kazunori Ogohara)
} 
(a)

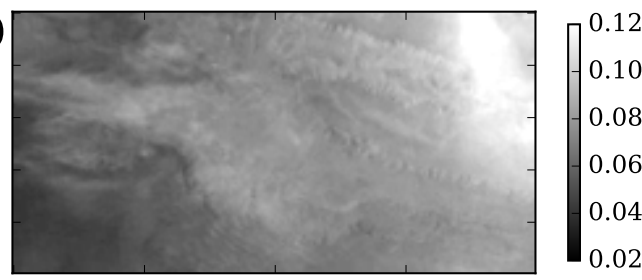

(c)

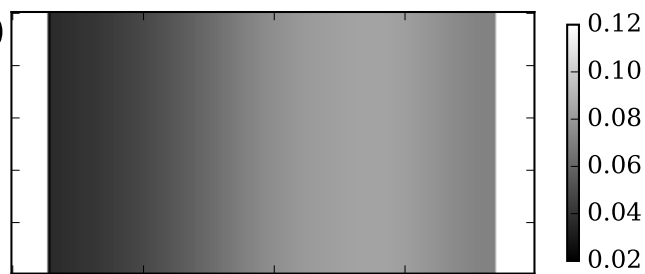

(b)

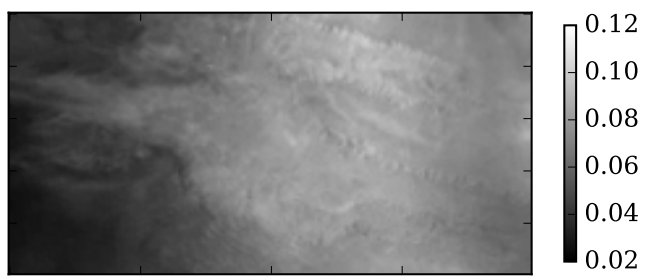

(d)

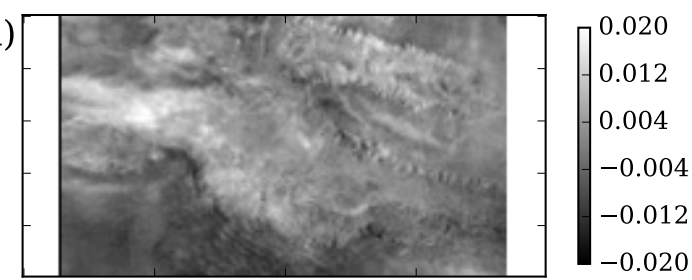

Figure 1: Sample images of the Valles Marineris in the blue band (a) before and (b) after the photometric correction proposed by Wang and Ingersoll (2002). (c), (b) smoothed using a running mean in the longitudinal direction. (d) A difference image obtained by subtracting (c) from (b). The image source is r1700109.imq (IMAGE_TIME $=$ 2004-05-01T23:48:35.75).

simulations that water ice clouds seen in the Tharsis result from water vapor advection by upslope wind (Spiga and Forget 2009). Visible images of Mars will continue to be used for studies on the morphology of water ice clouds and ice cloud parameterizations. Although water ice clouds are currently extracted visually from images in the visible wavelengths (Wang and Ingersoll, 2002), it clearly requires too much time to visually and individually check the growing number of images. Therefore, it is desirable to automatically estimate whether water ice clouds can be seen in an image of a target region.

Dust storms are another outstanding atmospheric phenomena. Radiatively active dust in the atmosphere scatters and absorbs solar radiance, and becomes a heat source. Dust storms have a dominant role in meteorology and in dust circulation in the Martian atmosphere. When we visually search for dust storms on visible images, we usually use blue band images (400-450 nm) as well as red band ones (575-625 nm) in order to distinguish dust storms from water ice clouds (Cantor et al., 2001). Therefore, a technique to automatically evaluate the presence of water ice clouds in blue images is useful not only for studies on Martian water circulation and the processes of ice cloud formation, but also for automated dust storm detection. Maeda et al. (2015) evaluated the existence of dust storms on MGS/MOC images in both wavelength ranges using high-dimensional feature vectors and sophisticated algorithms. However, a relationship between patterns recognized as a dust storm by the algorithm and physical properties of dust storms were not mentioned. Although their algorithms are useful as a tool for dust storm detection, it is difficult for meteorologists to obtain scientific information on dust storm properties using the aforementioned tool. Therefore, when detecting water ice clouds automatically, we require an algorithm that uses simple and easy features so that we can understand detected water ice clouds physically without decreasing the evaluation indices, as well as algorithms that pursue accuracy with complicated feature vectors. 
In this study, we aimed to automatically detect Martian water ice clouds with machine learning using visual inspection as a teacher data set. We did not adopt sophisticated high-dimensional feature vectors such as SIFT or HoG (e. g. Bandeira et al., 2011; Lin et al., 2014) but simple statistics that are easy to understand physically. We also found the two or three most effective features for evaluating the presence of water ice clouds, and will present the lowest detection accuracy that methods utilizing proven high-dimensional feature vectors have to achieve. In Section 2. we will describe the image data sets and pre-processing used. In Section 3, we will explain the chosen classifier and features for training the classifier. The results of the cross-validation will be shown in Section 4 . In Section 5 , features that are effective for the detection of water ice clouds will be investigated.

\section{Data and Pre-processing}

We used reflectance data (blue band, 400-450 nm) taken by MGS/MOC (Malin et al., 2010). In this study, we investigated methods for detecting water ice clouds in and around the Valles Marineris, where evidence that liquid water existed in the past have been found. The Valles Marineris is appropriate as a sample region for developing the detection algorithm for water ice clouds because it is a scientifically important site where the possibility of present life existing is increasing (Dohm and Miyamoto, 2016, Miyamoto et al., 2016). Blue band images of MOC were obtained through the U.S. Geological Survey (USGS, http://ida.wr.usgs.gov/d_access.htm). Noise reduction, radiometric calibration, and coordinate transformation to longitude-latitude coordinates were done using ISIS3 (https://isis.astrogeology.usgs.gov/index.html). The region of $5^{\circ} \mathrm{S}-15^{\circ} \mathrm{N}, 278^{\circ} \mathrm{E}-298^{\circ} \mathrm{W}$ where the Valles Marineris is located was trimmed from the longitude-latitude images.

Regional dust storms tend to occur frequently in both hemispheres after $L_{s}^{1}=140^{\circ}$ (Wang and Richardson, 2015). Even if an image includes both water ice clouds and dust storms, water ice clouds are expected to be selectively detected since the reflectance of dust storms in the blue band is small. However, to simplify the evaluation of the results, we focused on spring and summer in the northern hemisphere when few regional dust storms have been observed. Wang and Ingersoll (2002) reported that water ice clouds occur frequently and cover large areas in low latitudes and the highlands during the northern mid-summer (Wang and Ingersoll, 2002, Figure 2). During this season, the algorithm may output a high accuracy even if it concludes that water ice clouds exist on all images. Therefore, we instead focused on the season from $L_{s}=10^{\circ}$ to $50^{\circ} .131$ images of the Valles Marineris were obtained from images taken during this season in MY25 and MY26 (Fig. 11a). The image size is $200 \times 100$ pixels and the resolution is $0.1 \mathrm{deg}$ pixel $^{-1}$. Names of original image files and the observation dates are summarized in the supplementary file (SupplementaryTable1.xlsx).

The reflectance increases around regions where the phase angle is near $0^{\circ}$ (opposition surge). Opposition surge may be falsely recognized as water ice clouds because the opposition surge is very bright. We reduced the opposition

\footnotetext{
${ }^{1}$ Solar longitude. This indicates season on Mars. $L_{s}=0^{\circ}$ and $L_{s}=90^{\circ}$ mean the northern spring equinox and the northern summer solstice, respectively.
} 

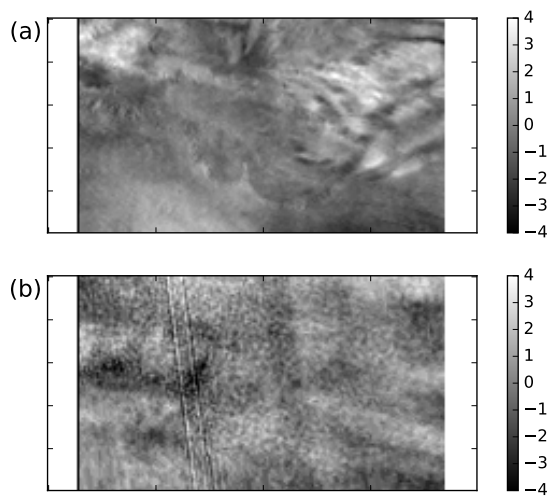

Figure 2: (a) An example of a subtracted image including water ice cloud signals. The source images were s1601476.img and s1700905.img. (b) An example of a subtracted image not including water ice cloud signals. The source images were m1801431.img and m1800987.img.

\subsection{Normalized subtracted image}

The water ice clouds that we tried to detect probably disappear or change their appearance drastically within a few sols. Although seasonal to inter-annual variation of the background pattern of the surface has been observed (Szwast et al. 2006; Fenton et al. 2007), the absolute value of the variation in a few sols can be negligible. Therefore, if there are water ice clouds on one of two blue images, the surface pattern is suppressed and water ice clouds should remain selectively on the difference image between the two images. Then, letting the two pre-processed images described in the previous section be $R_{1}$ and $R_{2}$, we can obtain the subtracted reflectance image $D=R_{2}-R_{1} . R_{2}$ is an image taken on a sol in which we evaluate the presence of water ice clouds. $R_{1}$ is another image taken on another sol as close to the sol as possible. The contrast and average of the subtracted images may be different from each other because opposition surge and large-scale reflectance patterns due to atmospheric aerosols may not be completely removed. Furthermore, it is preferable that the ice cloud detection algorithm we develop in this study is applicable to other regions and images taken by the Mars Color Imager onboard the Mars Reconnaissance Orbiter (MRO/MARCI). Therefore, we normalized 
a subtracted reflectance image on the basis of the following equation:

$$
D_{\mathrm{N}}=\frac{D-\bar{D}}{\sigma_{D}},
$$

where $\bar{D}$ and $\sigma_{D}$ is the average and standard deviation of $D$, respectively. The average and standard deviation in a normalized subtracted reflectance become 0 and 1 , respectively, for all subtracted images.

Figure 2 shows samples of normalized subtracted reflectance images. Figure $2 \mathrm{~h}$ is a normalized subtracted reflectance image generated by subtracting an image without water ice clouds from another image with water ice clouds. Figure $2 \mathrm{~b}$ shows the normalized reflectance difference between two images without water ice clouds. Water ice clouds are clearly seen in Fig. 2 a.

\subsection{Cross-correlation distribution}

In the cases where one of two images shows water ice clouds, the subtracted reflectance between the images increases. In contrast, it is expected that similarity between the two images will decrease. We calculated the crosscorrelation $r(i, j)$ at a pixel $(i, j)$ between $R_{1}$ and $R_{2}$ as follows:

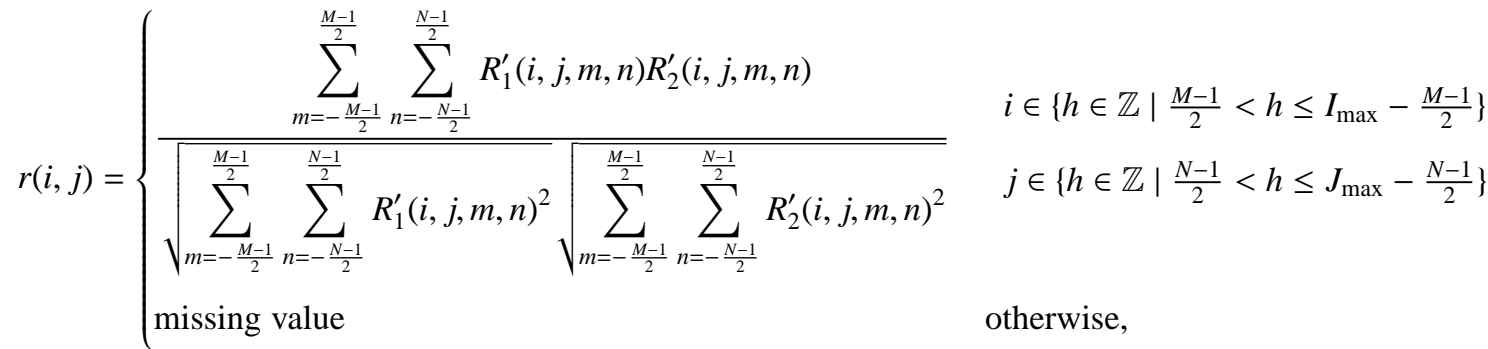

$$
\begin{aligned}
& R_{1}^{\prime}(i, j, m, n)=R_{1}(i+m, j+n)-\overline{R_{1}(i, j)}, \\
& R_{2}^{\prime}(i, j, m, n)=R_{2}(i+m, j+n)-\overline{R_{2}(i, j)}, \\
& \overline{R_{1}(i, j)}=\frac{1}{M N} \sum_{m=-\frac{M-1}{2}}^{\frac{M-1}{2}} \sum_{n=-\frac{N-1}{2}}^{\frac{N-1}{2}} R_{1}(i+m, j+n), \\
& \overline{R_{2}(i, j)}=\frac{1}{M N} \sum_{m=-\frac{M-1}{2}}^{\frac{M-1}{2}} \sum_{n=-\frac{N-1}{2}}^{\frac{N-1}{2}} R_{2}(i+m, j+n),
\end{aligned}
$$

where $I_{\max }$ and $J_{\max }$ are the number of columns and rows of a subtracted image. $M$ and $N$ are odd here. If $M$ and $N$ are small, small-scale clouds can be detected but the cross-correlation decreases sensitively to instrumental noise and local variations of the surface pattern, regardless of the presence of water ice clouds in the subtracted image. If $M$ and $N$ are large, small but evident features of water ice clouds fail to be detected. Both $M$ and $N$ were experimentally set to 21 in this study. Figure 3 indicates the cross-correlation distributions derived from the same source images as those used for Fig. 2. The cross-correlation in Fig. 3a seems to be less on average than that in Fig. 3 b.

We obtained one normalized subtracted image and one cross-correlation distribution from two images, $R_{1}$ and $R_{2}$, and calculated features from them. For simplicity, the features adopted in this study were the maximum $R_{\max }$, variance 

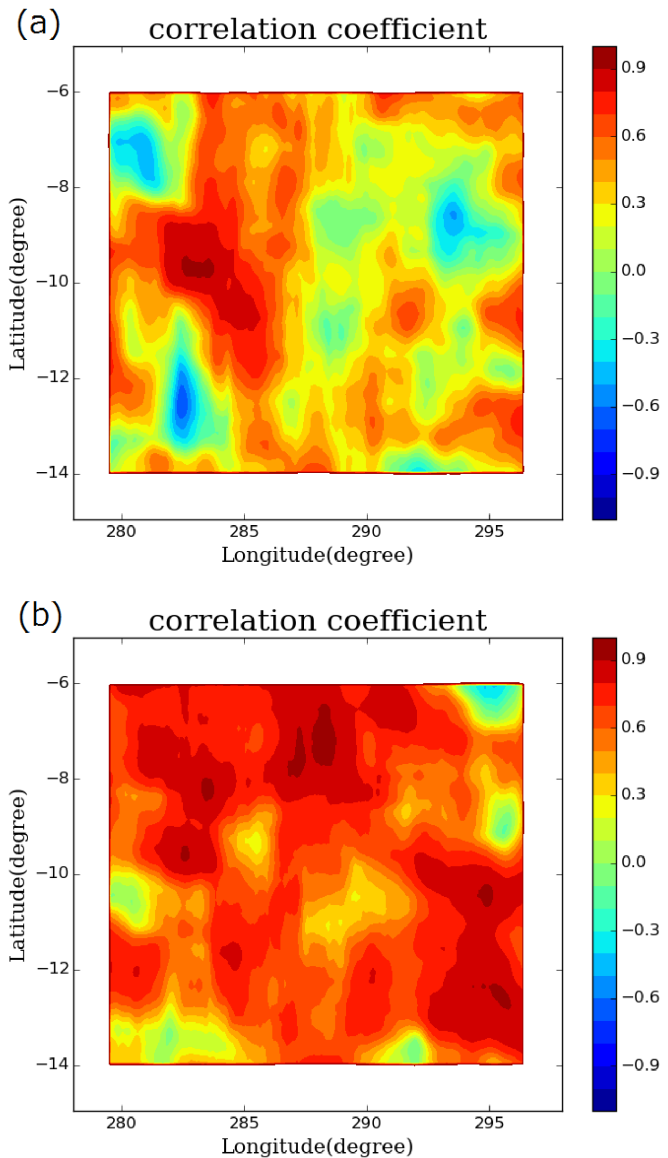

Figure 3: (a) An example of a cross-correlation distribution in the case where there are water ice cloud signals. The source images were the same as those of Fig. 2 2 . (b) An example of a cross-correlation distribution in the case where there is no water ice cloud signal. The source images were the same as those of Fig. 2 b.

$V$, kurtosis $K$, and skewness $S$ of the normalized subtracted reflectance, and the difference between the maximum and 
Table 1: Feature pairs for training the classifier. $R_{\max }, V, K$, and $S$ are the maximum, variance, kurtosis, and skewness of the normalized reflectance difference, respectively. $C_{\mathrm{mm}}, V_{\mathrm{C}}, K_{\mathrm{C}}$, and $S_{\mathrm{C}}$ are the difference between the maximum and average, variance, kurtosis and skewness of a crosscorrelation distribution, respectively.

\begin{tabular}{|c|c|c|c|c|c|c|c|c|c|c|c|c|c|c|c|}
\hline \multirow{2}{*}{ 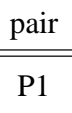 } & \multicolumn{3}{|c|}{ feature } & \multirow{2}{*}{$\begin{array}{l}\text { pair } \\
\text { P15 }\end{array}$} & \multicolumn{3}{|c|}{ feature } & \multicolumn{2}{|l|}{ pair } & \multicolumn{2}{|c|}{ feature } & \multirow{2}{*}{$\begin{array}{c}\text { pair } \\
\text { P43 }\end{array}$} & \multicolumn{3}{|c|}{ feature } \\
\hline & $R_{\max }$ & $S$ & $K$ & & $R_{\max }$ & $V$ & $S_{\mathrm{c}}$ & P29 & $S$ & V & $V_{\mathrm{c}}$ & & $K$ & $C_{\mathrm{mm}}$ & $S_{\mathrm{c}}$ \\
\hline $\mathrm{P} 2$ & $R_{\max }$ & $S$ & V & P16 & $R_{\max }$ & $C_{\mathrm{mm}}$ & $K_{\mathrm{c}}$ & P30 & $S$ & V & $S_{\mathrm{c}}$ & P44 & $K$ & $K_{\mathrm{c}}$ & $V_{\mathrm{c}}$ \\
\hline P3 & $R_{\max }$ & $S$ & $C_{\mathrm{mm}}$ & P17 & $R_{\max }$ & $C_{\mathrm{mm}}$ & $V_{\mathrm{c}}$ & P31 & $S$ & $C_{\mathrm{mm}}$ & $K_{\mathrm{c}}$ & P45 & $K$ & $K_{\mathrm{c}}$ & $S_{\mathrm{c}}$ \\
\hline $\mathrm{P} 4$ & $R_{\max }$ & $S$ & $K_{\mathrm{c}}$ & P18 & $R_{\max }$ & $C_{\mathrm{mm}}$ & $S_{\mathrm{c}}$ & P32 & $S$ & $C_{\mathrm{mm}}$ & $V_{\mathrm{c}}$ & P46 & $K$ & $V_{\mathrm{c}}$ & $S_{\mathrm{c}}$ \\
\hline P5 & $R_{\max }$ & $S$ & $V_{\mathrm{c}}$ & P19 & $R_{\max }$ & $K_{\mathrm{c}}$ & $V_{\mathrm{c}}$ & P33 & $S$ & $C_{\mathrm{mm}}$ & $S_{\mathrm{c}}$ & P47 & $V$ & $C_{\mathrm{mm}}$ & $K_{\mathrm{c}}$ \\
\hline P6 & $R_{\max }$ & $S$ & $S_{\mathrm{c}}$ & $\mathrm{P} 20$ & $R_{\max }$ & $K_{\mathrm{c}}$ & $S_{\mathrm{c}}$ & P34 & $S$ & $K_{\mathrm{c}}$ & $V_{\mathrm{c}}$ & P48 & $V$ & $C_{\mathrm{mm}}$ & $V_{\mathrm{c}}$ \\
\hline P7 & $R_{\max }$ & $K$ & $V$ & P21 & $R_{\max }$ & $V_{\mathrm{c}}$ & $S_{\mathrm{c}}$ & P35 & $S$ & $K_{\mathrm{c}}$ & $S_{\mathrm{c}}$ & P49 & $V$ & $C_{\mathrm{mm}}$ & $S_{\mathrm{c}}$ \\
\hline P8 & $R_{\max }$ & $K$ & $C_{\mathrm{mm}}$ & P22 & $S$ & $\mathrm{~K}$ & $V$ & P36 & $S$ & $V_{\mathrm{c}}$ & $S_{\mathrm{c}}$ & P50 & $V$ & $K_{\mathrm{c}}$ & $V_{\mathrm{c}}$ \\
\hline P9 & $R_{\max }$ & $K$ & $K_{\mathrm{c}}$ & P23 & $S$ & $\mathrm{~K}$ & $C_{\mathrm{mm}}$ & P37 & $K$ & V & $C_{\mathrm{mm}}$ & P51 & $V$ & $K_{\mathrm{c}}$ & $S_{\mathrm{c}}$ \\
\hline P10 & $R_{\max }$ & $K$ & $V_{\mathrm{c}}$ & P24 & $S$ & $\mathrm{~K}$ & $K_{\mathrm{c}}$ & P38 & $K$ & V & $V_{\mathrm{c}}$ & P52 & $V$ & $V_{\mathrm{c}}$ & $S_{\mathrm{c}}$ \\
\hline P11 & $R_{\max }$ & $K$ & $S_{\mathrm{c}}$ & P25 & $S$ & $\mathrm{~K}$ & $V_{\mathrm{c}}$ & P39 & $K$ & $V$ & $S_{\mathrm{c}}$ & P53 & $C_{\mathrm{mm}}$ & $K_{\mathrm{c}}$ & $V_{\mathrm{c}}$ \\
\hline $\mathrm{P} 12$ & $R_{\max }$ & $V$ & $C_{\mathrm{mm}}$ & P26 & $S$ & $\mathrm{~K}$ & $S_{\mathrm{c}}$ & P40 & $K$ & $C_{\mathrm{mm}}$ & $K_{\mathrm{c}}$ & P54 & $C_{\mathrm{mm}}$ & $K_{\mathrm{c}}$ & $S_{\mathrm{c}}$ \\
\hline $\mathrm{P} 13$ & $R_{\max }$ & $V$ & $K_{\mathrm{c}}$ & P27 & $S$ & V & $C_{\mathrm{mm}}$ & P41 & $K$ & $C_{\mathrm{mm}}$ & $K_{\mathrm{c}}$ & P55 & $C_{\mathrm{mm}}$ & $V_{\mathrm{c}}$ & $S_{\mathrm{c}}$ \\
\hline P14 & $R_{\max }$ & $V$ & $V_{\mathrm{c}}$ & P28 & $S$ & V & $K_{\mathrm{c}}$ & $\mathrm{P} 42$ & $K$ & $C_{\mathrm{mm}}$ & $V_{\mathrm{c}}$ & P56 & $S_{\mathrm{c}}$ & $K_{\mathrm{c}}$ & $V_{\mathrm{c}}$ \\
\hline
\end{tabular}

\subsection{Classifier}

Reflectance variations in the Valles Marineris are prominently visible on subtracted images. We assumed that the largest effect on reflectance in the blue band was caused by water ice clouds detached from the surface. However, in some cases, the entire interior of the Valles Marineris becomes brighter than the adjacent area (Fig. 4h). If an image where the Valles Marineris is relatively dark is subtracted from an image where the Valles Marineris is bright, a signal with the shape of the Valles Marineris is obvious on the subtracted reflectance image. Clouds or fog may possibly arise in the entirety of the Valles Marineris. However, studies using the Thermal Emission Spectrometer onboard MGS (MGS/TES) and numerical simulations are needed to investigate such lightening phenomena of the Valles Marineris, and are beyond the scope of this work. Therefore, the brightening of the Valles Marineris was eliminated from detection. We separated images with a bright valley and images with a dark valley before preparing subtracted images and prevented the Valles Marineris from brightening in subtracted images. Names of the source image files and the presence of water ice clouds are summarized in a supplementary file (SupplementaryTable2.xlsx). The presence or absence of water ice clouds was based on visual inspection by the authors. We categorized ambiguous images into the "without cloud" group. If we analyze MGS/TES data, we can possibly evaluate whether objects in subtracted images we regarded as water ice clouds were truly water ice clouds, dust storms, or noise. However, MGS/TES rarely simultaneously observes objects we regarded as water ice clouds in an image because the spatial 

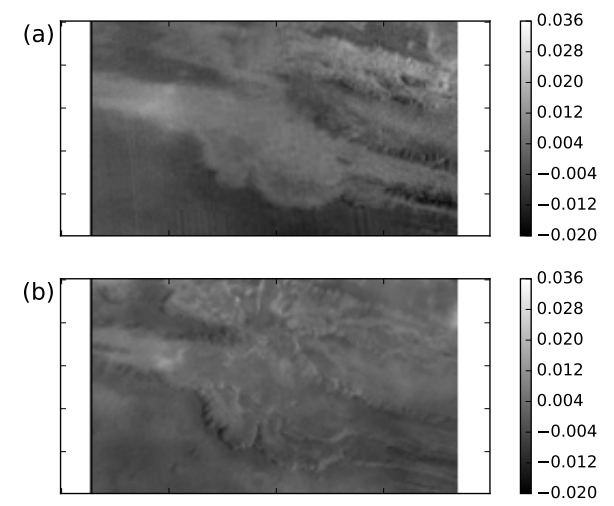

Figure 4: Corrected reflectance images of the target area in the cases of (a) a bright Valles Marineris and (b) a typical Valles Marineris. The image sources were m1700920.imq (IMAGE_TIME = 2000-07-27T04:13:28.76) and s1700905.imq (IMAGE_TIME = 2006-04-11T23:49:45.06), respectively.

\section{Results}

We evaluated the generalization ability of the classifier using 10-fold cross-validation. 130 image pairs were divided into ten groups and nine of them were used for training SVM. The other one was used for the test and the precision $P$, recall $R$, F-measure $F$, and accuracy $A$ were obtained. The training and test was repeated ten times for 
the different groups and the average of each evaluation index was calculated. $P, R, F$, and $A$ are defined as follows:

$$
\begin{aligned}
P & =\frac{\mathrm{TP}}{\mathrm{TP}+\mathrm{FP}} \\
R & =\frac{\mathrm{TP}}{\mathrm{TP}+\mathrm{FN}} \\
F & =\frac{2 P R}{P+R} \\
A & =\frac{\mathrm{TP}+\mathrm{TN}}{\mathrm{TP}+\mathrm{FP}+\mathrm{TN}+\mathrm{FN}}
\end{aligned}
$$

TP, TN, FP, and FN are the number of true positives, true negatives, false positives, and false negatives, respectively. The above procedure was repeated five times for different random groupings and the average of each evaluation index was obtained.

Figure 5 shows results of five 10-fold cross-validations for all combinations of three features. The pair with the largest $P$ was P53 (0.88, Fig. 51). Other pairs with a large P were P56 (0.85), P12 (0.84), P8 (0.84), P17 (0.84), P27 (0.84), and P42 (0.83). The pairs with the largest $R$ were P7 and P22 (1, Fig. 5b). Other pairs with a large $R$ were P2 (0.98), P25 (0.97), and P51 (0.95). Pairs with a large $P$ had a small $R$, and vice versa. In the cases of pairs with a large $P$ and a small $R(\mathrm{P} 53, \mathrm{P} 56, \mathrm{P} 12, \mathrm{P} 8, \mathrm{P} 17, \mathrm{P} 27$, and $\mathrm{P} 42)$, approximately $30 \%$ of the images where water ice clouds were visually identified were regarded as images without ice clouds. In the cases of pairs with a large $R$ and a small $P(\mathrm{P} 7, \mathrm{P} 22, \mathrm{P} 2, \mathrm{P} 25$, and P51), approximately $40 \%$ of images automatically judged to be positive showed no signal corresponding to water ice clouds, although more than $95 \%$ of images where water ice clouds were actually visible were successfully judged to be positives. If such pairs for which either $P$ or $R$ is large and the other is small are adopted as features, $F$ and $A$ tend to become small. The pairs with the largest $F$ were the same as those with the largest $A, \mathrm{P} 12, \mathrm{P} 17, \mathrm{P} 8$, and $\mathrm{P} 3$ (Figs. 56 and 5d). If both $P$ and $R$ are approximately $0.8, F$ and $A$ tend to become relatively large.

\section{Discussion and conclusion}

All pairs with a large $A(\mathrm{P} 3, \mathrm{P} 8, \mathrm{P} 12$, and $\mathrm{P} 17)$ included $R_{\max }$ and $C_{\mathrm{mm}}$ as features (Table 1). Other pairs including $R_{\max }$ and $C_{\mathrm{mm}}$ were P16 and P18 according to Table 1, The accuracy, $A$, of these two pairs, 0.74 and 0.75 , was comparable with $A$ of other pairs. Therefore, if both $R_{\max }$ and $C_{\mathrm{mm}}$ are used as features, a high accuracy is expected. In addition, the F-measure also tended to be high because pairs showing a large $F$ were approximately the same as those pairs showing a large $A$. However, $F$ was also high in the cases of P51 that included neither $R_{\max }$ nor $C_{\mathrm{mm}}$, and P30 which did not include $R_{\max }$. Although $V, S$, and $S_{\mathrm{C}}$ can be candidates for important features, their significance was not as clear as that of $R_{\max }$ and $C_{\mathrm{mm}}$. Figure 6 shows histograms of $F$ and $A$ displayed in Fig. 5 , $F$ and $A$ were clearly large in the cases where $R_{\max }$ and $C_{\mathrm{mm}}$ were used as features. The four pairs with the largest accuracy are especially outstanding.

It is easily physically understood that $R_{\max }$ and $C_{\mathrm{mm}}$ are useful for the automated detection of water ice clouds. Other than at the polar ice caps, reflectance in the blue band of the Martian surface is usually low. Reflectance in 

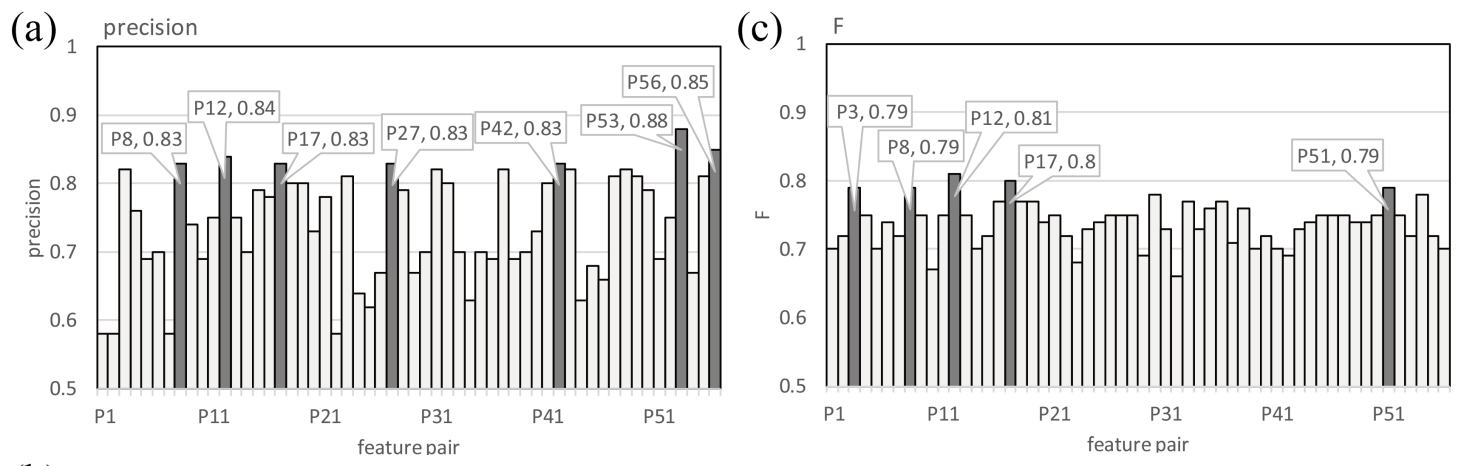

(b)

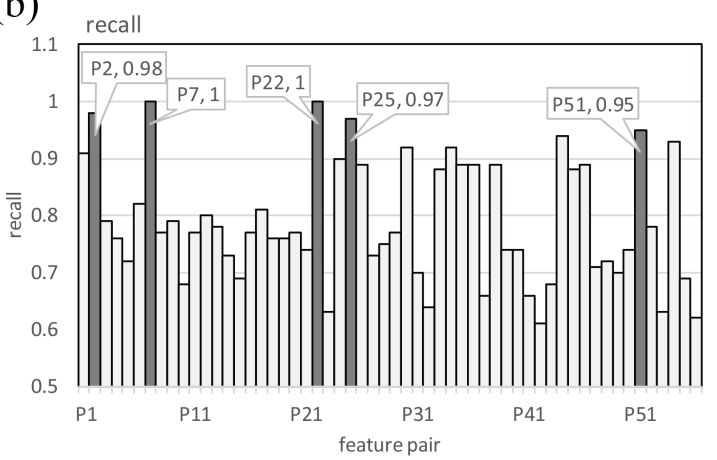

(d)

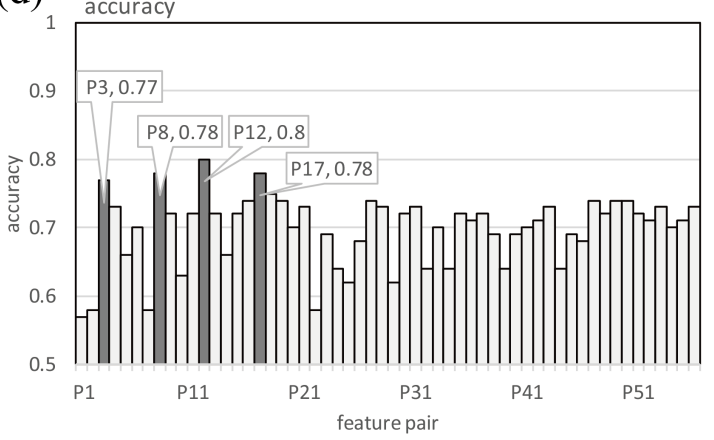

Figure 5: Averages in (a) precision, (b) recall, (c) F-measure, and (d) accuracy of each pair among five 10-fold cross validations.

the blue band of water ice clouds is larger than that of the surface (water ice clouds appear to be "white" in visible wavelengths). Therefore, areas where water ice clouds are obvious become bright in the subtracted reflectance. Since the surface patterns probably have no correlation with patterns of water ice clouds, cross-correlation becomes small in areas where water ice clouds are obvious, and then $C_{\mathrm{mm}}$ becomes large. These are qualitatively consistent with the fact that some pairs with large $F$ and $A$ include variance and skewness in cross-correlation.

We evaluated the presence or absence of Martian water ice clouds with SVM using images in visible wavelengths and cross-correlation distributions. The maximum $R_{\max }$, variance $V$, kurtosis $K$, and skewness $S$ of the normalized subtracted reflectance, and the difference between the maximum and average $C_{\mathrm{mm}}$, variance $V_{\mathrm{C}}$, kurtosis $K_{\mathrm{C}}$, and the skewness $S_{\mathrm{C}}$ of the cross-correlations were adopted as features for machine learning. Three of these eight features were used for training the classifier. We evaluated the generalization ability of the classifier using 10-fold cross-validation. When the maximum of the normalized subtracted reflectance, $R_{\max }$, and the difference between the maximum and average in cross-correlation, $C_{\mathrm{mm}}$, were used as features for training, an F-measure and an accuracy of $\sim 0.8$ were obtained. Each feature is a simple statistic and can be easily calculated from one image. Nevertheless, the results are comparable with those of the automated detection of dust storms on red and blue images using DSIFT and HoG (Maeda et al. 2015). Furthermore, the features that we used are much easier to understand physically than sophisticated feature vectors, and are appropriate as a benchmark for automated detection algorithms of water ice clouds and dust storms by machine learning using various feature vectors. 

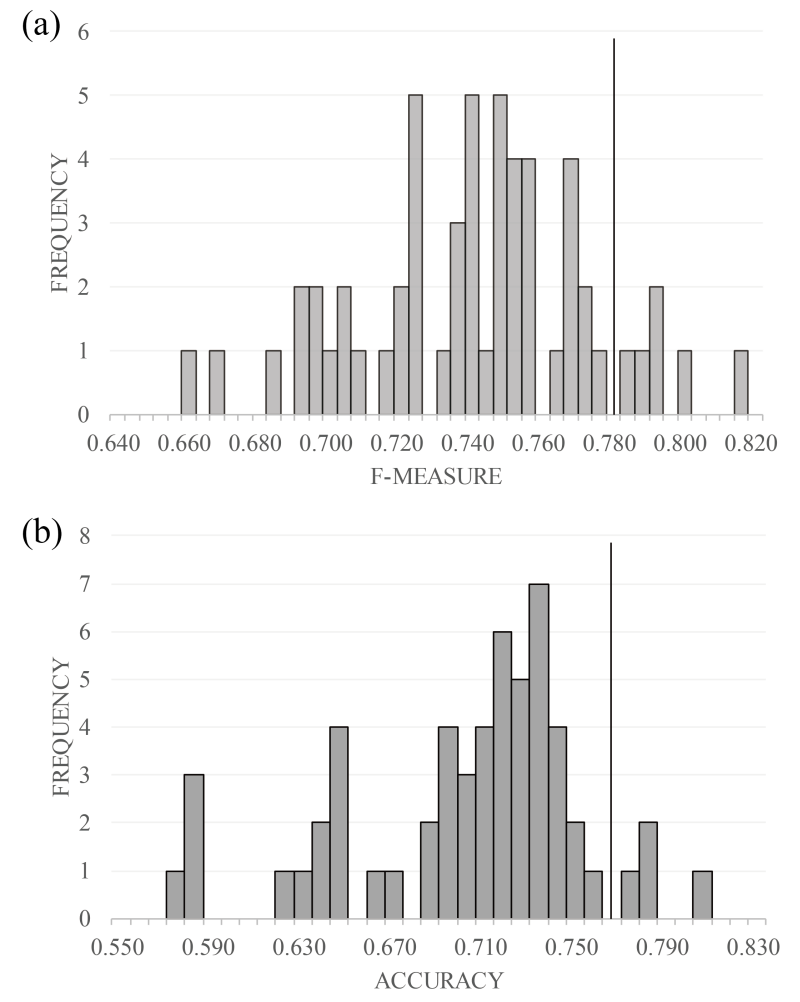

Figure 6: Histograms of (a) F-measure and (b) accuracy shown in Fig. 5

\section{Appendix}

Letting a two dimensional array whose the number of columns and rows were $X_{\max }$ and $Y_{\max }$, respectively, be $f$, variance $v$, skewness $s$ and kurtosis $k$ introduced in section 3.2 were defined as follows:

$$
\begin{aligned}
& v=\frac{1}{X_{\max } Y_{\max }-1} \sum_{i=1}^{X_{\max }} \sum_{j=1}^{Y_{\max }}\{f(i, j)-\bar{f}\}^{2} \\
& s=\frac{1}{X_{\max } Y_{\max }} \sum_{i=1}^{X_{\max }} \sum_{j=1}^{Y_{\max }} \frac{\{f(i, j)-\bar{f}\}^{3}}{\sigma_{f}^{3}} \\
& k=\frac{1}{X_{\max } Y_{\max }} \sum_{i=1}^{X_{\max }} \sum_{j=1}^{Y_{\max }} \frac{\{f(i, j)-\bar{f}\}^{4}}{\sigma_{f}^{4}}-3,
\end{aligned}
$$

where $\bar{f}$ and $\sigma_{f}$ were the average and the sample standard deviation of $f$, respectively. $v$ was unbiased variance. In section 3.2 $f$ was the normalized subtracted reflectance $D_{\mathrm{N}}$ when calculating $V, K$ and $S$. On the other hand, $f$ was 
the cross-correlation distribution defined by equation (2) when calculating $V_{\mathrm{C}}, K_{\mathrm{C}}$ and $S_{\mathrm{C}}$.

\section{References}

Bandeira, L., Marques, J.S., Saraiva, J., Pina, P., 2011. Automated detection of martian dune fields. IEEE Geosci. Remote Sens. Lett. 8, 626-630. doi 10.1109/LGRS. 2010.2098390

Cantor, B.A., James, P.B., Caplinger, M., Wolff, M.J., 2001. Martian dust storms: 1999 Mars Orbiter Camera observations. J. Geophys. Res. 106, 23653-87.

Dohm, J.M., Miyamoto, H., 2016. Geomorphological indication of ancient, recent, and possibly present-day aqueous activity on mars. J. Geogr. 125, 121-132. doi $10.5026 /$ jgeography.125.121

Fenton, L.K., Geissler, P.E., Haberle, R.M., 2007. Global warming and climate forcing by recent albedo changes on Mars. Nature 446, 646-9. URL: http://www.ncbi.nlm.nih.gov/pubmed/17410170 doi 10.1038/nature05718

Forget, F., Haberle, R.M., Montmessin, F., Levrard, B., Head, J.W., 2006. Formation of glaciers on Mars by atmospheric precipitation at high obliquity. Science 311,368-71. URL: http://www.ncbi.nlm.nih.gov/pubmed/16424337 doi 10.1126/science.1120335

Guzewich, S.D., Toigo, A.D., Kulowski, L., Wang, H., 2015. Mars Orbiter Camera climatology of textured dust storms. Icarus 258, 1-13. URL: http://dx.doi.org/10.1016/j.icarus.2015.06.023 doi 10.1016/j.icarus.2015.06.023

Ji, E.Y., Moon, Y.J., Park, J., Lee, J.Y., Lee, D.H., 2013. Comparison of neural network and support vector machine methods for kp forecasting. J. Geophys. Res.: Space Physics 118, 5109-5117. URL: http://dx.doi.org/10.1002/jgra.50500 doi 10.1002/jgra.50500

Lin, Y., Bunte, M., Saripalli, S., III, J.B., Greeley, R., 2014. Autonomous volcanic plume detection on planetary bodies. Acta Astron. 97,151 - 163. URL: http://www.sciencedirect.com/science/article/pii/S0094576513004323 doi 10.1016/j.actaastro.2013.11.029

Lu, J., Peng, Y., Wang, M., Gu, S., Zhao, M., 2016. Support vector machine combined with distance correlation learning for dst forecasting during intense geomagnetic storms. Planet. Space Sci. 120, 48 - 55. URL: http://www.sciencedirect.com/science/article/pii/ S0032063315003347 doi http://dx.doi.org/10.1016/j.pss.2015.11.004

Maeda, K., Ogawa, T., Haseyama, M., 2015. Automatic Martian Dust Storm Detection from Multiple Wavelength Data Based on Decision Level Fusion. IPSJ Transactions on Computer Vision and Applications 7, 79-83. doi 10.2197/ipsjtcva.7.79

Malin, M.C., Edgett, K.S., Cantor, B.A., Caplinger, M.A., Danielson, G.E., Jensen, E.H., Ravine, M.A., Sandoval, J.L., Supulver, K.D., 2010. An overview of the 1985-2006 Mars Orbiter Camera science investigation. Mars 5, 1-60. URL: http://www.mars journal.org/contents/ 2010/0001/ doi 10.1555/mars.2010.0001

Martín-Torres, F.J., Zorzano, M.P., Valentín-Serrano, P., Harri, A.M., Genzer, M., Kemppinen, O., Rivera-Valentin, E.G., Jun, I., Wray, J., Madsen, M.B., et al., 2015. Transient liquid water and water activity at gale crater on mars. Nature Geosci. .

Miyamoto, H., Komatsu, G., Dohm, J., Hemmi, R., Usui, T., Yamagishi, A., 2016. Geomorphological view of the environmental history of mars and candidate habitable environments. Journal of Geography 125, 171-184. doi 10.5026/jgeography.125.171

Montmessin, F., Forget, F., Rannou, P., Cabane, M., Haberle, R.M., 2004. Origin and role of water ice clouds in the Martian water cycle as inferred from a general circulation model. J. Geophys. Res. 109, 1-26. doi 10.1029/2004JE002284

Richardson, M.I., 2002. Investigation of the nature and stability of the Martian seasonal water cycle with a general circulation model. J. Geophys. Res. 107. URL: http://www.agu.org/pubs/crossref/2002/2001JE001536.shtml doi 10.1029/2001JE001536

Spiga, A., Forget, F., 2009. A new model to simulate the Martian mesoscale and microscale atmospheric circulation: Validation and first results. J. Geophys. Res. 114, 1-26. URL: http://www.agu.org/pubs/crossref/2009/2008JE003242.shtml doi 10.1029/2008JE003242

Szwast, M.A., Richardson, M.I., Vasavada, A.R., 2006. Surface dust redistribution on Mars as observed by the Mars Global Surveyor and Viking orbiters. J. Geophys. Res. 111. URL: http://www.agu.org/pubs/crossref/2006/2005JE002485.shtml doi 10.1029/2005JE002485 Wang, H., Ingersoll, A.P., 2002. Martian clouds observed by Mars Global Surveyor Mars Orbiter Camera. J. Geophys. Res. 107, 1-16. URL: http://www.agu.org/pubs/crossref/2002/2001JE001815.shtml doi 10.1029/2001JE001815

Wang, H., Richardson, M.I., 2015. The origin, evolution, and trajectory of large dust storms on Mars during Mars years 24-30(1999-2011). Icarus 251,112-127. URL:http://dx.doi.org/10.1016/j.icarus.2013.10.033 doi 10.1016/j.icarus.2013.10.033 
Wilson, R.J., Neumann, G.A., Smith, M.D., 2007. Diurnal variation and radiative influence of Martian water ice clouds. Geophys. Res. Lett. 34, 1-4. doi $10.1029 / 2006$ GL027976 